\title{
Efficacy of Intravenous Administration of Landiolol in Patients With Acute Heart Failure and Supraventricular Tachyarrhythmia
}

\author{
Shunsuke Kiuchia, b, Hiroto Aikawa ${ }^{a}$, Shinji Hisatake ${ }^{a}$, Takayuki Kabuki ${ }^{a}$, Takashi Oka ${ }^{a}$, \\ Shintaro Dobashi ${ }^{a}$, Takahiro Fujii ${ }^{\mathrm{a}}$, Takanori Ikeda ${ }^{\mathrm{a}}$
}

\begin{abstract}
Background: Patients with acute heart failure (HF) complicated by supraventricular tachyarrhythmia (SVT) often receive continuous intravenous infusion of landiolol or diltiazem for rate control. It is unclear whether the interval from initiation of infusion to commencement of oral beta-blocker (BB) therapy differs for these two drugs.
\end{abstract}

Methods: From January 2013 to July 2015, 94 consecutive patients were hospitalized for acute HF complicated by SVT. After 35 patients were excluded, the remaining 59 were divided into groups treated with diltiazem or landiolol. We investigated the blood pressure, heart rate, New York Heart Association classification, brain natriuretic peptide, chest X-ray film, echocardiographic findings (ejection fraction $(\mathrm{EF})$ ), time until commencement of oral BB therapy, and hospital stay.

Results: There were no significant between-group differences of heart rate, blood pressure, or the severity of HF. The time until commencing oral BB therapy was significantly shorter in the landiolol group compared with the diltiazem group (median: 2 vs. 4 days, $\mathrm{P}=$ 0.002 ), but there was no significant difference in hospital stay. This interval was significantly shorter in patients with a reduced $\mathrm{EF}$ in the landiolol group (median: 2 days) compared with those with a reduced $\mathrm{EF}$ in the diltiazem group (median: 5 days, $\mathrm{P}=0.008$ ), and patients with a preserved EF in the landiolol group tended to have a shorter interval (median: 2 days) than those with a preserved $\mathrm{EF}$ in the diltiazem group (median: 4 days, $\mathrm{P}=0.092$ ).

Conclusions: Switching to oral BBs was accomplished earlier with landiolol than with diltiazem.

Keywords: Beta-blocker; Calcium channel blocker; Hospital stay; Heart rate; Blood pressure

Manuscript accepted for publication March 01, 2017

aDepartment of Cardiovascular Medicine, Toho University Faculty of Medicine, Tokyo, Japan

${ }^{b}$ Corresponding Author: Shunsuke Kiuchi, Department of Cardiovascular Medicine, Toho University Faculty of Medicine, 6-11-1 Omori-nishi, Ota-ku, Tokyo 143-8541, Japan. Email: syunnsuke@med.toho-u.ac.jp

doi: https://doi.org/10.14740/jocmr2954w

\section{Introduction}

Oral beta-blockers (BBs) are an established standard treatment for chronic heart failure (HF) and prompt introduction of oral BB therapy is recommended for these patients [1]. In the USA and Europe, it has been reported that early introduction of oral $\mathrm{BBs}$ reduces the frequency of late cardiac events, and that patients starting oral BB therapy before discharge from hospital have lower mortality rate and readmission than patients starting oral BBs from 2 weeks after discharge [2]. A Japanese study has also shown that initiation of oral BB therapy before discharge from hospital significantly reduces the mortality rate and readmission [3]. Furthermore, it has been demonstrated that when intravenous medications of cardiac stimulants are used for the treatment of HF, early introduction of oral BB therapy is also beneficial [4]. Accordingly, guidelines recommend the introduction of low-dose oral BB therapy in the early stage of acute HF [5].

In Japan, there has been an increase in the prevalence rate of supraventricular tachycardia (SVT), including atrial fibrillation (AF) [6], and acute HF associated with SVT has also been increasing. For rate control, SVT patients often receive continuous intravenous infusion of the ultra-short-acting $\mathrm{BB}$ landiolol or the non-dihydropyridine calcium channel blocker (CCB) diltiazem. It has been unclear whether there is a difference between these two intravenous medications in the time required for subsequent switching to oral BB therapy. Therefore, we performed the present study to compare the time required for introduction of oral BBs in patients with acute HF and SVT receiving infusion of either landiolol or diltiazem.

\section{Methods}

\section{Subjects}

Among patients with acute HF (or acute exacerbation of chronic HF) accompanied by SVT who were inpatients of our hospital between January 1, 2013 and July 31, 2015, 94 consecutive patients receiving continuous infusion of landiolol or diltiazem were retrospectively investigated. Fifty-nine eligible patients were analyzed, after excluding 18 patients 
Table 1. Underlying Heart Diseases and Etiology of Supraventricular Tachycardia in Both Groups

\begin{tabular}{|c|c|c|c|c|}
\hline & Gri & $(n=44)$ & Gr & $(n=15)$ \\
\hline & HFrEF $(n=25)$ & HFpEF $(n=19)$ & HFrEF $(n=10)$ & HFpEF $(n=5)$ \\
\hline Tachycardia-induced cardiomyopathy (n) & 15 & 12 & 5 & 1 \\
\hline Ischemic cardiomyopathy (n) & 3 & 0 & 2 & 2 \\
\hline Dilated cardiomyopathy (n) & 1 & 0 & 0 & 0 \\
\hline Atrial flutter (n) & 1 & & 0 & \\
\hline
\end{tabular}

There were no significant differences of other findings between the two groups, and intergroup differences were not found after stratification by HFrEF and HFpEF. HFrEF: heart failure with a reduced ejection fraction; HFpEF: heart failure with a preserved ejection fraction.

using oral BBs before hospitalization, 13 patients switched from landiolol or diltiazem to other intravenous medications for rate control, one patient treated with landiolol for ventricular tachycardia (VT), and three patients who died in hospital. Comparison was performed between group D treated with diltiazem $(\mathrm{n}=44)$ and group $\mathrm{L}$ treated with landiolol $(\mathrm{n}=15)$.

\section{Study outcomes}

The clinical endpoint of the present study was the interval until initiation of oral BBs from the start of continuous intravenous infusion of landiolol or diltiazem for rate control after hospitalization. While the necessity for rate control during acute phase of HF has been demonstrated, there is no generally accepted target. Therefore, we manage our HF patients with SVT by setting the target heart rate (HR) at $\leq 110 /$ min based on previous reports [7]. After the target HR is achieved, the patient is switched to oral BB therapy as soon as possible while maintaining rate control and avoiding aggravation of $\mathrm{HF}$. In the present study, we also investigated the efficacy of each drug for achieving the J-LAND study target (HR at $2 \mathrm{~h}$ after commencing intravenous infusion $\leq 110 /$ min or less than $80 \%$ of baseline) [8]. Furthermore, we determined the total duration of hospital stay in addition to the time until commencement of oral BB therapy. Moreover, we investigated the severity of HF based on the New York Heart Association (NYHA) classification, laboratory findings, chest X-ray findings, and transthoracic echocardiography (TTE) findings. Patients with HF can be classified into those who have HF with a reduced ejection fraction (HFrEF) and those who have HF with a preserved ejection fraction (HFpEF), and the pathogenesis of $\mathrm{HF}$ and re-hospitalization rates differ between these two groups [9]. Therefore, we also conducted a separate comparison of HFrEF and HFpEF patients. In the present study, we defined HFrEF as an $\mathrm{EF}<50 \%$ on TEE [10].

\section{Clinical profile}

We assessed the underlying heart disease and the type of SVT.
We also investigated the HR and systolic/diastolic blood pressure (BP) before commencement and $2 \mathrm{~h}$ after the start of infusion. We assessed the starting dose of oral BB therapy, and the dose at discharge from hospital. Doses of oral BBs were converted to bisoprolol equivalent values.

\section{Chest X-ray film}

The cardiothoracic ratio (CTR) was calculated by two physicians using the maximal cardiac diameter and the intrathoracic diameter on a chest X-ray film obtained in the standing position on admission.

\section{TTE}

TTE was performed to evaluate cardiac size (left atrial dimension and left ventricular end-diastolic/end-systolic dimensions) and systolic function (EF and percent fractional shortening). The EF was calculated by the modified Simpson's method [11].

\section{Statistical analysis}

Results were presented as the median or the mean \pm standard deviation. We used the Mann-Whitney U test, the Wilcoxon single-rank test, or the paired $t$-test for comparisons between group L and group D, as well as for assessing the changes in $\mathrm{BP}$ and HR. The Chi-square test was used to compare underlying diseases. Analyses were performed with Microsoft Excel and the Stat View statistical package (Stat View 4.0, SAS Institute Inc.). A probability (P) value of less than 0.05 was considered to indicate statistical significance.

\section{Ethical considerations}

The present study was approved by the Toho University Omori Medical Center Ethical Committee on September 12, 2012 (approval no.: 24-123). All experiments were performed in ac- 
cordance with the Declaration of Helsinki.

\section{Results}

\section{Clinical profile}

The clinical profile of the patients is summarized in Table 1. There were no significant differences of underlying heart diseases between the two groups. In addition, no intergroup differences were found after stratification of each group into HFrEF and HFpEF subgroups.

In group D, SVT was due to AF in 43 patients and by atrial flutter in one patient (who had HFrEF), while the etiology of SVT was AF in all 15 patients from group L. There was no significant difference in the cause of SVT between the two groups.

\section{Baseline characteristics on admission}

Table 2 shows the clinical characteristics of the subjects on admission. There were no significant differences in the laboratory findings, CTR, and TTE parameters. Significant intergroup differences were also not seen after stratification into HFrEF and HFpEF subgroups.

\section{Initiation of oral BB therapy}

Although the time until initiation of oral BB therapy was significantly shorter in group $\mathrm{L}$ than group $\mathrm{D}$ (4 days and 2 days, $\mathrm{P}=0.002)$, there was no significant difference in hospital stay between the two groups (Fig. 1). The time until commencement of oral BB therapy was also significantly shorter for HFrEF patients from group $\mathrm{L}$ than group $\mathrm{D}$, and it tended to be shorter for HFpEF patients (Fig. 2). Oral BB therapy was initiated during hospitalization in all patients $(100 \%)$ from group L and in $93.2 \%$ of patients from group D (not initiated in three patients). The initial dose of the oral BB (bisoprolol equivalent) was $0.625 \mathrm{mg}$ /day in group D and $0.625 \mathrm{mg} /$ day in group $\mathrm{L}(\mathrm{P}=0.897)$, while the dose at discharge from hospital was $2.500 \mathrm{mg} /$ day in group D and $2.500 \mathrm{mg}$ /day in group L $(\mathrm{P}=0.760)$. There were also no significant differences when the patients were stratified into subgroups with HFrEF or HFpEF. Among patients with HFrEF, the initial dose was $0.625 \mathrm{mg} /$ day in both groups $(\mathrm{P}=0.762)$, while the dose at discharge was $1.563 \mathrm{mg}$ /day in group D and 2.500 $\mathrm{mg}$ /day in group $\mathrm{L}(\mathrm{P}=0.385)$. Among patients with $\mathrm{HFpEF}$, the initial dose was $0.625 \mathrm{mg} /$ day in both groups $(\mathrm{P}=0.557)$ and the dose at discharge was $2.500 \mathrm{mg} /$ day in both groups $(\mathrm{P}=0.784)$.

\section{HR and BPs}

HR decreased significantly in both groups after the start of infusion (Table 3). BP also showed a significant decrease in
Table 2. Characteristics, Laboratory Findings, CTR and Echocardiographic Findings of the Subjects

\begin{tabular}{|c|c|c|c|}
\hline & $\begin{array}{l}\text { Group D } \\
(n=44)\end{array}$ & $\begin{array}{l}\text { Group L } \\
(n=15)\end{array}$ & $\mathbf{P}$ \\
\hline Age (years) & 77 & 74 & 0.292 \\
\hline Male/female & $17 / 27$ & $10 / 5$ & 0.107 \\
\hline Height (cm) & 159.0 & 165.0 & 0.077 \\
\hline Weight (kg) & 52.7 & 55.8 & 0.139 \\
\hline BMI $\left(\mathrm{kg} / \mathrm{m}^{2}\right)$ & 20.3 & 21.6 & 0.577 \\
\hline NYHA class & 3 & 3 & 0.787 \\
\hline Sodium $(\mathrm{mEq} / \mathrm{L})$ & 140 & 140 & 0.525 \\
\hline Potassium (mEq/L) & 3.9 & 4.0 & 0.370 \\
\hline Albumin (g/dL) & 3.6 & 3.6 & 0.870 \\
\hline Total bilirubin (mg/dL) & 1.0 & 1.1 & 0.447 \\
\hline AST (IU/L) & 35 & 41 & 0.583 \\
\hline ALT (IU/L) & 28.0 & 30 & 0.938 \\
\hline LDH (IU/L) & 304 & 295 & 0.737 \\
\hline BUN (mg/dL) & 21.5 & 18.0 & 0.086 \\
\hline Creatinine (mg/dL) & 0.96 & 0.84 & 0.161 \\
\hline eGFR (mL/min/1.73 m²) & 49.8 & 55.2 & 0.074 \\
\hline Hemoglobin (g/dL) & 13.1 & 14.5 & 0.338 \\
\hline $\mathrm{BNP}(\mathrm{pg} / \mathrm{mL})$ & 605.8 & 767.6 & 0.754 \\
\hline CTR (\%) & 61.9 & 63.6 & 0.389 \\
\hline LAD (mm) & 44 & 37 & 0.186 \\
\hline LVDd (mm) & 50 & 48 & 0.741 \\
\hline LVDs (mm) & 39 & 42 & 0.572 \\
\hline $\mathrm{EF}(\%)$ & 47 & 42 & 0.554 \\
\hline$\%$ FS (\%) & 24 & 20 & 0.486 \\
\hline
\end{tabular}

There were no significant differences between the two groups. BMI: body mass index; NYHA: New York Heart Association; AST: aspartate aminotransferase; ALT: alanine aminotransferase; LDH: lactate dehydrogenase; BUN: blood urea nitrogen; eGFR: estimated glomerular filtration rate; BNP: brain natriuretic peptide; CTR: cardiothoracic ratio; LAD: left atrial dimension; LVDd: left ventricular end-diastolic dimension; LVDs: left ventricular end-systolic dimension; EF: ejection fraction; \%FS percent fractional shortening.

group D after $2 \mathrm{~h}$ of infusion (systolic BP: $131.1 \pm 23.7 \mathrm{~mm} \mathrm{Hg}$ to $121.1 \pm 20.3 \mathrm{~mm} \mathrm{Hg}, \mathrm{P}<0.001$; diastolic BP: $81.4 \pm 19.0$ $\mathrm{mm} \mathrm{Hg}$ to $69.9 \pm 13.3 \mathrm{~mm} \mathrm{Hg}, \mathrm{P}<0.001)$. In contrast, there was no significant decrease in BP in group L (Table 3).

Infusion of diltiazem or landiolol was started at 0.5 or 1.0 $\mu \mathrm{g} / \mathrm{kg} / \mathrm{min}$, and the infusion rate was gradually increased while monitoring BP and HR. The maximum infusion rate was 2.65 $\pm 1.16 \mu \mathrm{g} / \mathrm{kg} / \mathrm{min}$ for diltiazem and $5.57 \pm 4.78 \mu \mathrm{g} / \mathrm{kg} / \mathrm{min}$ for landiolol. In group L, eight out of 15 patients achieved the target HR set in the J-LAND study, which was a significantly higher percentage than in group $\mathrm{D}(14 / 44, \mathrm{P}=0.009)$. However, there was no significant difference between landiolol and diltiazem after stratification of the patients into HFpEF and HFrEF subgroups (HFpEF: group D 3/19, group L 3/5, P = 
Duration of hospital stay

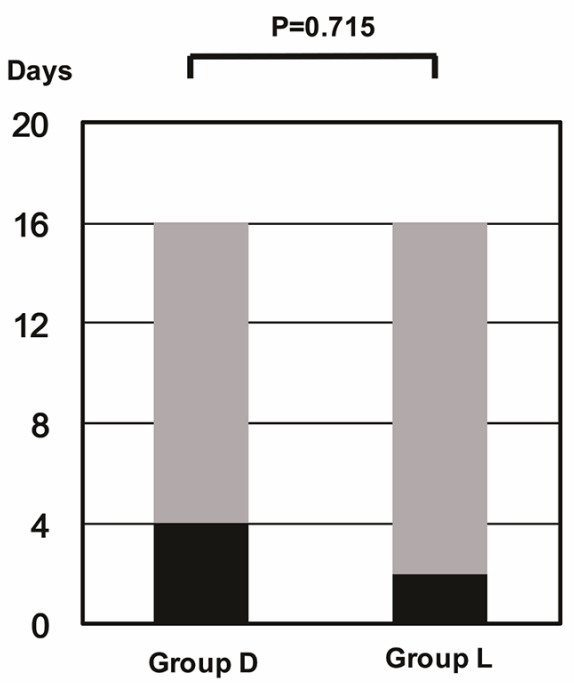

Time until initiation of oral betablocker therapy

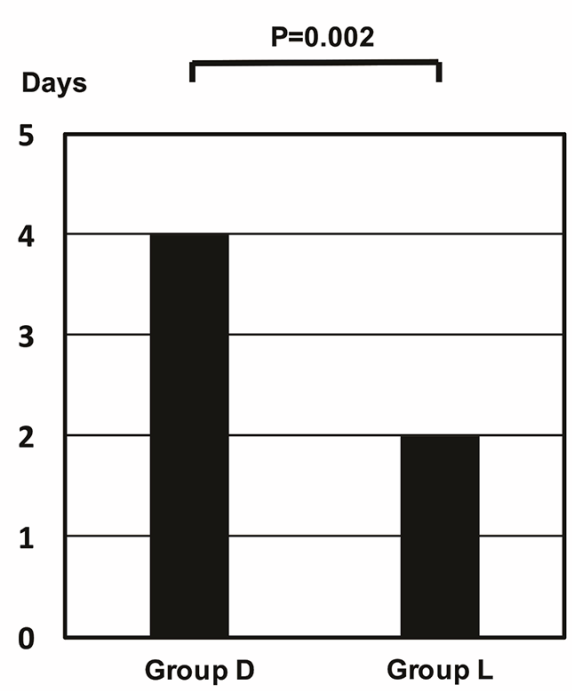

Figure 1. Time until initiation of oral beta-blocker therapy. The time was significantly longer in group $D$ than in group $L$.

0.136; HFrEF: group D 11/25, group L 5/10, P=0.708).

\section{Discussion}

\section{Main findings}

In the present study, the groups treated with landiolol and diltiazem showed no significant differences in patient charac- teristics, underlying heart disease, or the type of SVT. There were also no significant differences in the severity of HF on admission. However, the interval until initiation of oral BB therapy was significantly shorter in group L than group D. On the other hand, there was no significant difference in the duration of hospitalization, indicating that an early transition to oral BB therapy did not either shorten or prolong hospitalization. Early switching from infusion of intravenous medications to oral BBs may allow patients to become more active and shorten the time in bed, possibly leading to prevention of mus-

Time until initiation of oral beta-blocker therapy (HFrEF)

Time until initiation of oral beta-blocker therapy (HFpEF)
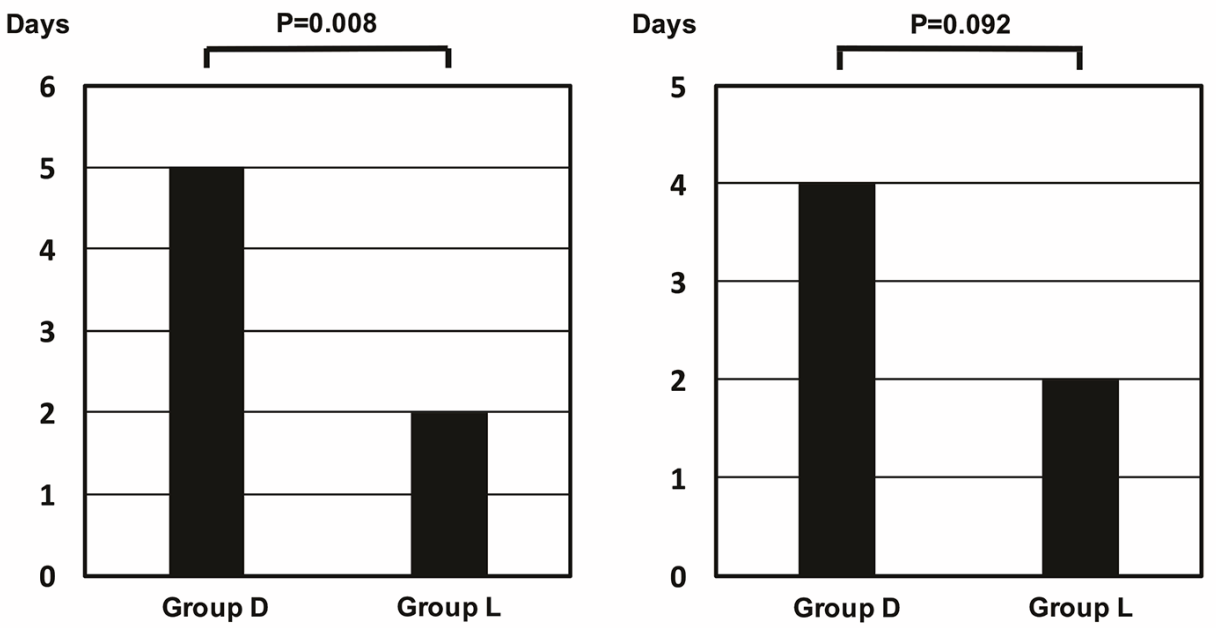

Figure 2. Time until initiation of oral beta-blocker therapy in patients stratified by ejection fraction. The time was significantly longer in patients with a reduced ejection fraction from group $D$ than group $L$. It was also longer in patients with preserved ejection fraction from group D, but the difference was not significant. HFpEF: heart failure with a preserved ejection fraction; HFrEF: heart failure with a reduced ejection fraction. 
Table 3. Blood Pressure and Heart Rate

\begin{tabular}{clll}
\hline & Baseline & After 2 h & P \\
\hline Group D ( $\mathrm{n}=44)$ & & & \\
SBP (mm Hg) & $131.1 \pm 23.7$ & $121.1 \pm 20.3$ & $<0.001$ \\
DBP (mm Hg) & $81.4 \pm 19.0$ & $69.9 \pm 13.3$ & $<0.001$ \\
HR (bpm) & $135.6 \pm 19.1$ & $111.1 \pm 26.1$ & $<0.001$ \\
Group L (n = 15) & & & \\
SBP (mm Hg) & 116 & 112 & 0.534 \\
DBP (mm Hg) & 70 & 64 & 0.443 \\
HR (bpm) & 132 & 98 & $<0.001$ \\
\hline
\end{tabular}

BP was significantly reduced after administration only in group D. SBP systolic blood pressure; DBP: diastolic blood pressure; HR heart rate.

cle weakness.

\section{Rate control therapy with BBs}

For rate control in patients with SVT, BBs, non-dihydropyridine $\mathrm{CCBs}$, or digitalis are selected depending on systolic function of heart. With digitalis preparations, the blood concentration has a narrow therapeutic range, and there is a risk of digitalis intoxication, especially in patients with reduced renal function. It has been suggested that using digitalis may increase the mortality rate [12], and BBs or non-dihydropyridine CCBs are often selected. While BBs have already been demonstrated to improve the prognosis of HFrEF patients [6], CCBs have not. Therefore, BBs are selected more frequently and it is considered useful to administer as high a dose as possible in patients with HF [3]. All of this evidence has been obtained for oral BBs, which means that oral agents should be introduced at an early stage and titrated until an adequate dose is reached. In many patients, SVT was treated by catheter-based myocardial ablation, which made it difficult to assess the long-term prognosis. However, switching to oral BBs was accomplished earlier with landiolol than with diltiazem.

\section{BP in both groups}

Both diltiazem and landiolol significantly reduced the HR, but the target HR of the J-LAND study was reached in a significantly higher proportion of patients from group L. While both of these agents are useful for suppressing tachycardia, there is a marked difference in their negative inotropic effect. Non-dihydropyridine CCBs are known to have a relatively strong negative inotropic effect and there is concern that this may reduce BP, and the American Heart Association/American College of Cardiology guidelines exclude CCBs from the medications recommended for heart dysfunction and failure [13]. Landiolol is not considered to reduce the BP, as was also shown in the present study. It has been reported that landiolol does not reduce $\mathrm{BP}$ in patients with $\mathrm{AF}$ [14] or in patients with sepsis who have a predisposition to low BP [15]. Avoiding a significant decrease in BP would have contributed to earlier switching to oral BBs in group L. However, this was a retrospective study and there was a BP difference of approximately $11 \mathrm{~mm} \mathrm{Hg}$ between the groups at the start of administration. The choice between landiolol and diltiazem was made by the attending physician, suggesting that landiolol which does not cause hypotension may have been chosen for patients with a lower baseline BP. In the future, a prospective double-blind study will be required to compare landiolol and diltiazem more accurately.

\section{Pleiotropic effects of BBs}

BBs have an antiarrhythmic effect. A hospital-based study of patients with $\mathrm{HF}$ reported that oral BBs reduced the occurrence of AF by $27 \%$ [16]. In addition to oral formulations, it has been reported that intravenous landiolol normalizes sinus rhythm in patients with AF after thoracotomy [17] and can be safely used for rate control [18]. In the present study, reversion to sinus rhythm was not achieved by intravenous therapy. Because it was achieved by cardioversion or catheter-based myocardial ablation in some patients after discharge, it was difficult to assess the efficacy of medication alone. Many clinical trials have demonstrated that oral BBs have a preventive effect on sudden cardiac death in patients with HF [19]. This may be partly due to suppression of VT, but it is noteworthy that this effect has also been reported with landiolol [20]. Such patients were excluded from the present study (one patient with VT received landiolol).

BBs have been reported to display pleiotropic effects. An increase of pro-inflammatory cytokines and oxidative stress has been demonstrated in AF [21], while carvedilol shows an anti-inflammatory effect and has been reported to inhibit oxidative stress. Additionally, oral BBs to suppress sympathetic activity and the renin angiotensin aldosterone system inhibitors as well as inhibiting apoptosis, myocardial fibrosis, and calcium overload $[22,23]$. While the actions of each BB are slightly different, bisoprolol and carvedilol both achieved similar outcomes in patients with HF [24]. However, reports of these pleiotropic effects are based on studies of oral BBs and the effects of intravenous BBs require further investigation in the future.

\section{Study limitations}

This was a retrospective study conducted in a small group of patients. The statistically significant results might not reflect a true effect and should be interpreted carefully. Treatment with landiolol or diltiazem was selected by the attending physician, and the baseline BP of the patients and differences in cost (landiolol is more expensive than diltiazem) may have influenced the choice of medication. Similarly, the timing and method of switching to an oral BB, as well as the dose escalation regimen, were determined by the attending physician. The second limitation is that we excluded patients who were not switched directly to oral BBs from intravenous landiolol or diltiazem. All of the patients treated with diltiazem who had not been 
receiving oral $\mathrm{BBs}$ on admission switched directly to oral $\mathrm{BB}$, except those who died in hospital. However, some of the patients treated with landiolol were switched to other intravenous medications and were excluded from the present study. Most of these patients had exacerbation of HF associated with low BP. Despite its effectiveness, landiolol is not well tolerated by some patients, and care should be exercised when determining whether to continue using this medication. Identifying patients for whom landiolol is appropriate therapy remains a subject for future research.

\section{Conclusions}

In patients with HF and SVT, switching from intravenous landiolol to oral BBs could be performed sooner than switching from intravenous diltiazem. Because our investigation was a retrospective study performed in a small patient population at a single site, a multicenter study needs to be conducted in a larger number of patients to confirm the present findings. Since HF was sometimes exacerbated by landiolol, care must be taken when selecting patients for infusion of this medication.

\section{Disclosure}

This research was financially supported by Ono Pharmaceutical, Co., Ltd. The company was not involved in study design, in the collection, analysis, and interpretation of data, in the writing of the report, or in the decision to submit this paper for publication. TI has received grant support through his institution from Daiichi Sankyo, Bristol-Myers Squibb, and Boehringer Ingelheim, and honoraria for lectures from Bayer Healthcare, Daiichi Sankyo, Bristol-Myers Squibb, Pfizer, Mitsubishi Tanabe Pharma, and Ono Pharmaceutical. The other authors have no conflicts of interest to disclose.

\section{References}

1. de Groote P, Delour P, Mouquet F, Lamblin N, Dagorn J, Hennebert O, Le Tourneau T, et al. The effects of betablockers in patients with stable chronic heart failure. Predictors of left ventricular ejection fraction improvement and impact on prognosis. Am Heart J. 2007;154(3):589595.

2. Gattis WA, O'Connor CM, Gallup DS, Hasselblad V, Gheorghiade M. Predischarge initiation of carvedilol in patients hospitalized for decompensated heart failure: results of the Initiation Management Predischarge: Process for Assessment of Carvedilol Therapy in Heart Failure (IMPACT-HF) trial. J Am Coll Cardiol. 2004;43(9):15341541.

3. Tsuchihashi-Makaya M, Kinugawa S, Yokoshiki H, Hamaguchi S, Yokota T, Goto D, Goto K, et al. Betablocker use at discharge in patients hospitalized for heart failure is associated with improved survival. Circ J. 2010;74(7):1364-1371.

4. Krum H, Roecker EB, Mohacsi P, Rouleau JL, Tendera M,
Coats AJ, Katus HA, et al. Effects of initiating carvedilol in patients with severe chronic heart failure: results from the COPERNICUS Study. JAMA. 2003;289(6):712-718.

5. Guidelines for Treatment of Acute Heart Failure (JCS 2011). http://www.j-circ.or.jp/guideline/pdf/JCS2011 izumi_h.pdf (in Japanese). Accessed 31 January 2017.

6. Yamashita Y, Hamatani Y, Esato M, Chun YH, Tsuji H, Wada H, Hasegawa K, et al. Clinical Characteristics and Outcomes in Extreme Elderly (Age $>/=85$ Years) Japanese Patients With Atrial Fibrillation: The Fushimi AF Registry. Chest. 2016;149(2):401-412.

7. Van Gelder IC, Groenveld HF, Crijns HJ, Tuininga YS, Tijssen JG, Alings AM, Hillege HL, et al. Lenient versus strict rate control in patients with atrial fibrillation. $\mathrm{N}$ Engl J Med. 2010;362(15):1363-1373.

8. Nagai R, Kinugawa K, Inoue H, Atarashi H, Seino Y, Yamashita T, Shimizu W, et al. Urgent management of rapid heart rate in patients with atrial fibrillation/flutter and left ventricular dysfunction: comparison of the ultrashort-acting beta1-selective blocker landiolol with digoxin (J-Land Study). Circ J. 2013;77(4):908-916.

9. Hamaguchi S, Kinugawa S, Sobirin MA, Goto D, Tsuchihashi-Makaya M, Yamada S, Yokoshiki H, et al. Mode of death in patients with heart failure and reduced vs. preserved ejection fraction: report from the registry of hospitalized heart failure patients. Circ J. 2012;76(7):16621669.

10. Marui A, Nishiwaki N, Komiya T, Hanyu M, Tanaka S, Kimura T, Sakata R. Comparison of 5-Year Outcomes After Coronary Artery Bypass Grafting in Heart Failure Patients With Versus Without Preserved Left Ventricular Ejection Fraction (from the CREDO-Kyoto CABG Registry Cohort-2). Am J Cardiol. 2015;116(4):580-586.

11. Schiller NB, Shah PM, Crawford M, DeMaria A, Devereux R, Feigenbaum $\mathrm{H}$, Gutgesell $\mathrm{H}$, et al. Recommendations for quantitation of the left ventricle by two-dimensional echocardiography. American Society of Echocardiography Committee on Standards, Subcommittee on Quantitation of Two-Dimensional Echocardiograms. J Am Soc Echocardiogr. 1989;2(5):358-367.

12. Adams KF, Jr., Butler J, Patterson JH, Gattis Stough W, Bauman JL, van Veldhuisen DJ, Schwartz TA, et al. Dose response characterization of the association of serum digoxin concentration with mortality outcomes in the Digitalis Investigation Group trial. Eur J Heart Fail. 2016;18(8):1072-1081.

13. January CT, Wann LS, Alpert JS, Calkins H, Cigarroa JE, Cleveland JC, Jr., Conti JB, et al. 2014 AHA/ACC/ HRS guideline for the management of patients with atrial fibrillation: a report of the American College of Cardiology/American Heart Association Task Force on Practice Guidelines and the Heart Rhythm Society. J Am Coll Cardiol. 2014;64(21):e1-76.

14. Kobayashi S, Murakami W, Myoren T, Tateishi H, Okuda S, Doi M, Nao T, et al. A low-dose beta1-blocker effectively and safely slows the heart rate in patients with acute decompensated heart failure and rapid atrial fibrillation. Cardiology. 2014;127(2):105-113.

15. Okajima M, Takamura M, Taniguchi T. Landiolol, an 
ultra-short-acting beta1-blocker, is useful for managing supraventricular tachyarrhythmias in sepsis. World J Crit Care Med. 2015;4(3):251-257.

16. Nasr IA, Bouzamondo A, Hulot JS, Dubourg O, Le Heuzey JY, Lechat P. Prevention of atrial fibrillation onset by beta-blocker treatment in heart failure: a meta-analysis. Eur Heart J. 2007;28(4):457-462.

17. Sakamoto A, Kitakaze M, Takamoto S, Namiki A, Kasanuki H, Hosoda S. Landiolol, an ultra-short-acting beta(1)-blocker, more effectively terminates atrial fibrillation than diltiazem after open heart surgery: prospective, multicenter, randomized, open-label study (JL-KNIGHT study). Circ J. 2012;76(5):1097-1101.

18. Kinugawa K, Nagai R, Inoue H, Atarashi H, Seino Y, Yamashita T, Shimizu W, et al. Impacts of patient characteristics on the effectiveness of landiolol in AF/AFL patients complicated with LV dysfunction: Subgroup analysis of the J-Land study. Adv Ther. 2014;31(4):426-439.

19. Siddiqui A, Kowey PR. Sudden death secondary to cardiac arrhythmias: mechanisms and treatment strategies. Curr Opin Cardiol. 2006;21(5):517-525.

20. Miwa Y, Ikeda T, Mera H, Miyakoshi M, Hoshida K,
Yanagisawa $\mathrm{R}$, Ishiguro $\mathrm{H}$, et al. Effects of landiolol, an ultra-short-acting beta1-selective blocker, on electrical storm refractory to class III antiarrhythmic drugs. Circ J. 2010;74(5):856-863.

21. Okada A, Kashima Y, Tomita T, Takeuchi T, Aizawa K, Takahashi M, Ikeda U. Characterization of cardiac oxidative stress levels in patients with atrial fibrillation. Heart Vessels. 2016;31(1):80-87.

22. Singh K, Xiao L, Remondino A, Sawyer DB, Colucci WS. Adrenergic regulation of cardiac myocyte apoptosis. J Cell Physiol. 2001;189(3):257-265.

23. Doi M, Yano M, Kobayashi S, Kohno M, Tokuhisa T, Okuda S, Suetsugu M, et al. Propranolol prevents the development of heart failure by restoring FKBP12.6mediated stabilization of ryanodine receptor. Circulation. 2002;105(11):1374-1379.

24. Hori M, Nagai R, Izumi T, Matsuzaki M. Efficacy and safety of bisoprolol fumarate compared with carvedilol in Japanese patients with chronic heart failure: results of the randomized, controlled, double-blind, Multistep Administration of bisoprolol IN Chronic Heart Failure II (MAINCHF II) study. Heart Vessels. 2014;29(2):238-247. 